\title{
Energy Efficient Interest Forwarding in NDN-Based Wireless Sensor Networks
}

\author{
Shuai Gao, ${ }^{1}$ Hongke Zhang, ${ }^{1}$ and Beichuan Zhang $^{2}$ \\ ${ }^{1}$ School of Electronic and Information Engineering, Beijing Jiaotong University, Beijing 100044, China \\ ${ }^{2}$ The University of Arizona, Tucson, AZ 85721, USA
}

Correspondence should be addressed to Shuai Gao; gaoxlh@gmail.com

Received 7 January 2016; Accepted 10 March 2016

Academic Editor: Xiaohong Jiang

Copyright (C) 2016 Shuai Gao et al. This is an open access article distributed under the Creative Commons Attribution License, which permits unrestricted use, distribution, and reproduction in any medium, provided the original work is properly cited.

Recently there has been a new emerging trend in integrating Named Data Networking (NDN) and wireless sensor networks (WSNs) together to implement real data-centric Internet of Things (IoT). However, the main solutions in current literature lack energy efficient design to meet the severely limited energy resources in WSNs. In this paper, we propose a dual mode Interest forwarding scheme (called DMIF in short) for NDN-based WSNs. The DMIF consists of two combined forwarding modes, in which several energy efficient mechanisms including flexible mode shift, flooding scope control, broadcast storm avoidance, packet suppression, and energy weight factors are designed to save and balance the energy consumption. We extend the ndnSIM to support wireless multihop communication to validate the proposed scheme. Simulation experiments show that the DMIF outperforms the baseline schemes in terms of total energy consumption, energy equilibrium rate, and network lifetime.

\section{Introduction}

Starting as a futuristic concept, the idea of wireless sensor networks (WSNs) has come a long way in past decades. Meantime, routing design is always one of the most important research topics in WSNs community $[1,2]$. In earlier stage of WSNs research, data-centric routing protocols play important roles, in which the sink sends Interests to the network and waits for data from the sensors without awareness of the identity of the sensors in advance. The basic idea of the data-centric routing is decoupling the sensed content from the sensor identity. However, such data-centric schemes are based on stand-alone solutions for routing [3], rather than a comprehensive solution for the whole Internet and Internet of Things (IoT). In addition, there are no detailed and standard naming schemes for various and complex applications in the literature [1]. All these factors limit the deployment of the data-centric approaches in practice for WSNs.

Fortunately, as an emerging future Internet architecture, Named Data Networking (NDN) [4] has been proposed and developed based on information centric networking in recent years. The NDN adopts a receiver-based service model and changes the communication paradigm from traditional host-centric communication to named data-centric communication. In the NDN, forwarding of Interest and Data packets is performed based on a distributed way, rather than centralized name resolution and content discovery, which naturally meets the requirements of WSNs and IoT well. The emergence of the NDN provides a good opportunity to rethink how to design real data-centric WSNs. Recently, some research works have been investigated on how to apply the NDN to WSNs $[3,5-9]$ and how the WSNs fit much more naturally into the emerging NDN architecture compared to conventional TCP/IP based technologies.

Nevertheless, there are still some open issues left for research [10], including naming, routing and forwarding, security, caching, transport control, and deployment mode, since original NDN is designed aiming at a whole Internet architecture, rather than WSNs.

In this paper, we focus on Interest forwarding and routing issues for NDN-based WSNs. Regarding routing and forwarding, there have been few works in this area. In the literature [3], a routing scheme inspired by the directed diffusion (DD) [11] and coupled with several anticollision timers is proposed to meet the requirements of periodical monitoring applications. In [5], the authors design a one-hop forwarding 
and routing protocol for multisource data retrieval in NDNbased IoT. A vanilla Interest flooding scheme and a reactive optimistic name-based routing scheme are proposed in [6]. However, these proposed routing schemes work without consideration about controlled flooding scope and energy awareness, so that they are not suitable for WSNs with severely limited energy and computation resources.

In addition, some research works [10] have dealt with forwarding and routing schemes in NDN-based wireless ad-hoc networks. Due to the challenges on the mobility and resource limitation in ad hoc networks, reactive flooding is usually adopted to discover potential content providers, rather than proactive routing, which needs too much cost to maintain the routing reachability for wireless ad hoc networks.

According to the survey in [10], the reactive forwarding in named data wireless ad hoc networks can be classified into two categories: blind forwarding and aware forwarding. In the blind forwarding, each node broadcasts Interest packets to discover the content sources. To avoid broadcast storm and improve source discovery efficiency, some timer-based packet suppression schemes $[12,13]$ are proposed to control the blind broadcast of Interest forwarding. On the other hand, the basic idea of the aware forwarding is selecting the next-forwarders [14-16] or content providers [12, 17, 18] in the Interest forwarding based on awareness of related network information, such as transmission distance, message redundancy, geographical location, and data retrieval rate. However, all the previous solutions about the Interest forwarding are based on wireless ad hoc networks and lack of considerations about severely limited energy resources and more challenging environment in WSNs.

In this paper, based on previous works in the literature, we propose an energy efficient dual mode Interest forwarding approach (named DMIF in short) for NDN-based WSNs. The DMIF consists of two forwarding modes: flooding mode and directive mode. In the flooding mode, three schemes including scope control, packet suppression, and broadcast storm avoidance based on an energy weight factor are designed to support controlled flooding and reduce the network overhead. In the directive mode, the Interest forwarding is guided in an energy awareness way based on the lookup results at the Forwarding Information Base (FIB) maintained by the passing Data packets along the reverse paths, thus improving the Interest forwarding and content transfer efficiency. The two modes in the DMIF work in a combined way and mode shift is allowed at any relay nodes if necessary. In addition, we extend ndnSIM $[19,20]$ to support wireless multihop communication and conduct simulation experiments, the results of which validate the proposed scheme and show that the DMIF scheme can reduce and balance total energy consumption and prolong network lifetime significantly in contrast with the baseline schemes.

The remainder of this paper is organized as follows. Section 2 reviews the related work. Section 3 describes the concerned application scenarios and a three-dimensional naming scheme. Section 4 is devoted to a detailed design of the energy efficient Interest forwarding based on two combined modes. Section 5 presents our simulation results and Section 6 concludes the paper.

\section{Related Work}

2.1. NDN: An Overview. As one promising ICN architecture, NDN [4] replaces the IP addresses with content chunks at the narrow waist of current Internet's hourglass model. A receiver-driven and content centric communication paradigm is defined in the NDN-based on the exchange of two types of packets: Interest and Data, which carries hierarchically structured names. Each NDN router maintains three data structures: (i) a FIB indexed by the content name prefixes and used to guide the Interest packets to the content sources; (ii) a Pending Interest Table (PIT) storing all Interests that are not yet satisfied and guiding the Data packets along the reverse path; (iii) a content store (CS) which temporarily caches the incoming Data packets to satisfy future Interests.

To retrieve a content, a consumer sends an Interest packet carrying the name of the desired content into the network. When a NDN router receives an Interest from a downstream neighbor, it makes a lookup in local CS by matching the content name at first. If the match hits, the router will send the Data back towards the consumer. If the match in the CS fails and a match in the PIT is found, the Interest will be discarded. Otherwise, a new PIT entry will be created and the Interest will be further forwarded to the upstream neighbor(s) based on the FIB information. When a NDN router receives Data, it forwards the Data along the reverse path taken by the Interest based on the PIT. Meanwhile, the router caches the content carried in the Data packet and the related PIT entries are removed. Thus, the Data packet returns to the requesting consumer finally.

In general, the NDN uses different interfaces at a node to discriminate the upstream neighbors and downstream neighbors. In the NDN, a node can forward the incoming Interest to the upstream neighbors by sending out the packet over all the network interfaces except the one from which the Interest arrives. In wireless multihop communication environment considered in this paper, there is only single wireless network interface at a node, which poses a challenge in the design of the Interest forwarding strategy for wireless NDN.

2.2. NDN-Based Wireless Sensor Networks. The receiverbased service model in the NDN fits naturally into datacentric WSNs. Recently, some research works [3, 5-9] have shown that WSNs can benefit from basic idea of the NDN design.

In [7], a wireless recharging system for WSNs is proposed based on the NDN to validate that the hierarchical naming structure fits in naturally with energy aggregation requirements and its inherent mobility supporting ability is very attractive for mobile recharging system. In order to meet the memory and computational constraints of WSNs, a light-weight content centric networking protocol [8] with simplified message format and flexible naming strategy is proposed for WSNs. In [9], the authors analyze the benefits (e.g., security, data aggregation, and mobility) by applying the NDN into IoT applications and provide a high-level NDN architecture that specially meets the IoT challenges. However, there are no Interest forwarding strategies for WSNs discussed in the above literature [7-9]. 
In [5], a comprehensive framework for reliable retrieval from multiple wireless sources is proposed based on Data suppression, collision avoidance, and Exclude field carried in the Interest packets. However, the proposed framework is only suitable for one-hop wireless scenarios. Interest forwarding based on multihop mesh networks is discussed in $[3,6]$. A blind flooding based on a deferred timer is designed to discover potential content providers in [3]. In addition, inspired by the DD protocol [11], each NDN node maintains a direction state during the Data packet forwarding in the blind flooding phase to facilitate future Interest forwarding. Similarly, a vanilla Interest forwarding (VIF) and a reactive optimistic name-based routing (RONR) are proposed in [6] to support basic blind and stateful forwarding, respectively. In our paper, the proposed DMIF combines two Interest forwarding modes together and supports flexible mode shift when needed, which is different from the related two Interest forwarding schemes in $[3,6]$ working in a parallel way. In addition, energy consumption and controlled scope are integrated into the blind forwarding phase to improve the energy efficiency of the system in this paper. In [21], the authors extended the deferred timer calculation in [3] to let the nodes with higher energy level forward Interest packets with higher priority. However, there is no weight factor between residual energy and transmission distance considered in the timer calculation. Moreover, the energy efficient stateful forwarding is not supported in [21].

2.3. Interest Forwarding in NDN-Based Wireless Ad Hoc Networks. Compared with WSNs, more research works have been investigated in NDN-based wireless ad hoc networks, mostly focusing on vehicular communications. According to the survey in [10], the reactive forwarding in named data wireless ad hoc networks can be classified into two categories: blind forwarding and aware forwarding.

In the blind forwarding, some timer-based packet suppression schemes $[12,13]$ are proposed to control the blind broadcast of the Interest forwarding and avoid the broadcast storm. In [13], the authors develop a collision prevention solution based on a uniform random timer to support NDNbased $\mathrm{V} 2 \mathrm{~V}$ traffic information dissemination. In addition, a proactive data pushing scheme through active overhearing is proposed in [13] to speed up the Data propagation, which is different from the receiver-based service model in original NDN design. Similarly, a set of timers and packet suppression solutions are proposed in $[12,17,18]$ to avoid packet collision in NDN-based vehicular environment. The deferred timer for Interest is longer than the one for Data, which gives higher access priority to Data over Interest packets. The above blind forwarding schemes are based on uniform random timers, whereas the deferred timer in this paper is based on transmission distance and residual energy at intermediate nodes and makes the flooding-based forwarding not too blind.

On the other hand, the basic idea of the aware forwarding is selecting the next-forwarders [14-16] or content providers $[12,17,18]$ in the Interest forwarding based on the awareness of related network information, such as transmission distance, message redundancy, geographical location, and data retrieval rate. In [14], a direction-selective forwarding scheme is proposed to choose the farthest node in each quadrant as the forwarder by information exchanging among Interest senders and receivers with two extended types of messages. The dedicated information exchanging for choice of the relay nodes may increase the system complexity. In the proposed DMIF, the deferred timer based on transmission distance gives the farther nodes with higher priority in the Interest forwarding without extra information exchanging. In the neighborhood-aware Interest forwarding (NAIF) scheme [15], the relay nodes are chosen in a probabilistic way based on two important historical factors, data retrieval rate and forwarding rate. The former is the ratio of the number of Data packets successfully retrieved to the number of Interest packets sent, and the latter reflects the fraction of the incoming Interest packets that a given node will forward. In [16], a gossip algorithm named BlooGo is developed to disseminate messages throughout the wireless NDN network with a minimum number of transmissions. The basic idea is that a relay node forwards the message only if its neighborhood is not strictly included into the one of the sender. The comparison of the neighboring relationship is performed based on a bloom filter. In a word, the above aware forwarding schemes need extra algorithms to obtain the related information for choice of the relay nodes, whereas the aware forwarding in the DMIF is based on the information left by the passing Data packets without extra overhead.

In the literature $[12,17,18]$, the authors design a provideraware forwarding (PAF) scheme to guide the Interest forwarding after the blind flooding. Each node maintains a distance table recording the distance information between local node and potential content providers. After receiving multiple Data packets replied from potential content providers, the consumers choose the nearest one among them and carry the provider ID and distance information in future Interest packets. The relay nodes forward the Interests to the direction of the selected provider based on the distance table. The basic idea in $[12,17,18]$ is similar to the proposed DMIF. In our paper, we are concerned about energy efficient forwarding for Interest packets. Specifically, the hop and residual energy metrics are carried in Data packets along the reverse paths to maintain the FIB and assist in future Interest forwarding in order to balance energy consumption and prolong network lifetime. In addition, the provideraware forwarding and blind forwarding are two parallel modes in $[12,17,18]$, which means the content consumer needs to restart the blind forwarding after the provider-aware forwarding fails in the relay nodes. In the DMIF, the two modes work in a combined way and mode shift is allowed at any relay nodes when needed.

\section{Scenario Description and Naming Scheme}

In this paper, we are concerned about a NDN-based WSN application in which multiple sinks are acting as content consumers that send periodic Interests to query the related information (e.g., temperature, humidity) sensed by the static sensors acting as content providers in specific areas. The sinks are powerful devices, but the sensor nodes are resource limited devices, including energy, computing, and memory, 

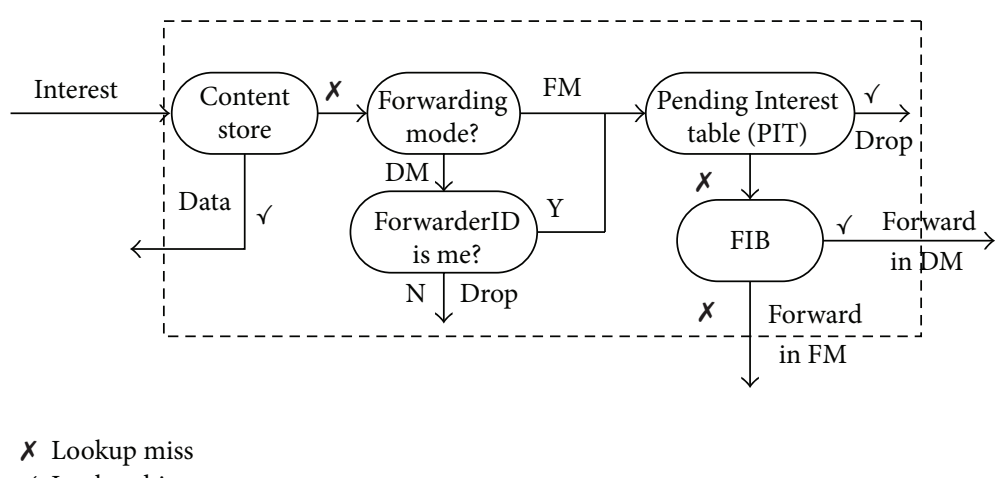

FIGURE 1: Dual mode Interest forwarding for NDN-based WSNs.

requiring that the process of the content query and retrieval should be energy efficient.

Data naming is one of the most important technologies in the NDN architecture, which may affect the design of the Interest forwarding and routing. In the concerned application, there are three-dimensional attributes to describe a content name: time dimension, space dimension, and type dimension. Therefore, based on the readable and hierarchical rules of NDN naming, we propose the following threedimensional naming for the raw data in this application, in which "WSNs" is a dedicated prefix to identify the proposed naming scheme.

$$
\text { /WSNs/type/.../space/.../time/... }
$$

(i) Type: type of the information sensed by the deployed sensor nodes, for example, "/type/temperature", "/type/humidity."

(ii) Space: the location attribute of the sensed content. It can be expressed as natural granularity, like a building ID or a room number. Another way is using a granularity configured manually for more general purpose. For example, we can divide the monitored area into several subareas based on the requirements of the application. And we use the subarea ID to describe the attribute of space, like "/space/areaA." We assume that each node knows where it locates and the content name of the raw data generated locally.

(iii) Time: the granularity in the time dimension is 1 minute in this paper. In other words, the raw data is generated every minute. Therefore, the format of the time dimension is expressed as "/time/date/minute index."

(a) Date: we use the format "/year/month/day/," for example, "/2015/12/04."

(b) Minute_index: $1,2, \ldots, n, \ldots, 1440$; here, $n$ denotes the time period $\{n-1, n\}$ minute within one day. For example, “/2015/12/04/1000" denotes the time period from $16: 39$ to $16: 40$ on December 4, 2015.
The above naming format consists of three components \{space, time, type\} that describe the content from three parallel dimensions. For each component, a hierarchical name is used to support flexible granularity and can be aggregated freely. The naming scheme here provides a recommended format. The order and the granularity of the three dimensions can be adjusted based on system requirements in practice.

\section{Dual Mode Interest Forwarding (DMIF)}

In this paper, we propose a dual mode Interest forwarding strategy based on the following considerations.

(1) Reactive routing is more efficient than proactive rout ing in resource limited WSNs based on NDN [22]. Therefore, flooding mode is the simplest way to discover the potential content providers.

(2) The flow of Data packets along the reverse paths leaves a trail of "breadcrumbs" [23], which can help find the content sources more quickly at less cost. Based on this, another Interest forwarding mode, directive mode, may be adopted to guide the Interest forwarding more efficiently.

The above two modes are not two parallel modes, which can be integrated together into the NDN forwarding plane and shifted to each other according to the FIB lookup results.

In the proposed DMIF, a content consumer checks the FIB at first before sending out an Interest packet. If the FIB lookup hits successfully, the Interest will be sent in the directive mode (DM). Otherwise, the Interest will be sent in the flooding mode (FM). In the DM mode, an extended field (named as ForwarderID) about the unique ID of the nexthop forwarder is carried in the Interest packet to designate a neighbor node as the forwarder of the Interest. A node receiving the Interest packet will check local content store as specified in original NDN design. If the content store lookup fails, the node will perform Interest forwarding based on the proposed DMIF illustrated in Figure 1. If the incoming Interest is in the FM mode, the node should continue the Interest forwarding if there are no duplicated Interests received before. If the incoming Interest is in the DM mode 


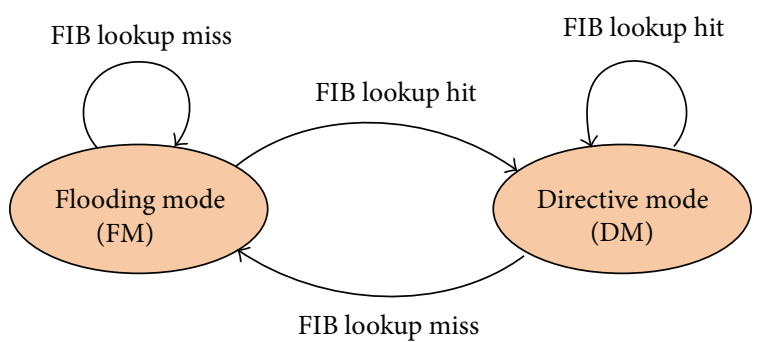

FIGURE 2: Interest forwarding mode shift in the DMIF scheme.

and the node is the same as the one designated by the ForwarderID field, the node will perform the forwarding. Regarding the forwarding, a node needs to check local FIB and choose suitable forwarding mode shown in Figure 1 based on the results of the FIB lookup.

Note that it is not guaranteed that single mode like the FM or the DM can be performed for Interest forwarding in an end-to-end path. During the forwarding in the DM mode, the FIB lookup at an intermediate node may fail due to some uncertain reasons, like node failure or entry timeout. If so, the DM mode can shift to the FM mode directly. In the literature $[12,17,18]$, if there is a lookup miss in an intermediate node, the content consumer needs to choose another content provider or choose to flood Interest after the Interest times out. In the DMIF, the two Interest forwarding modes can be combined together and mode shifts are allowed at any intermediate nodes when needed based on the FIB lookup results shown in Figure 2.

4.1. Flooding Mode (FM). In case of FIB lookup miss, the flooding mode (FM) is used to disseminate the Interest packets. In the FM mode, the content consumer or the intermediate forwarders broadcast the Interest packets through the wireless link to discover potential content provider(s). The key point of the FM mode is how to implement controlled flooding to reduce energy consumption and improve discover efficiency. Here, three schemes are adopted to support controlled flooding: scope control, broadcast storm avoidance, and packet suppression.

4.1.1. Scope Control. Interest flooding without controlled scope will continue until it goes throughout the network arriving at the network edge, even if the content providers have already been discovered. Uncontrolled flooding will cause severe waste of bandwidth and energy resources.

To address this issue, a new field about TTL (Time To Live) is added into the Interest packet to control the dissemination scope of an Interest. Firstly, the Interest is flooded with the TTL values set to TTL_START. The TTL value is reduced by 1 at each forwarding node in the FM mode. If the Interest times out, the content consumer will increase the TTL value by TTL_INCREMENT to restart the content discovery process. The choice of TTL_START and TTL_INCREMENT is dependent on the network scale and performance requirements on energy consumption or discover delay.
4.1.2. Broadcast Storm Avoidance. In related literature, a deferred timer is often adopted in the Interest flooding to avoid or alleviate the effects of broadcast storm. The deferred timer is generally based on a random counter, which lacks enough control on the path selection. In wireless sensor networks, unbalanced energy consumption may lead to energy depletion too early and shorten the network lifetime. Here, we design an energy efficient Interest flooding scheme with a deferred timer based on transmission distance and residual energy.

Upon receiving an Interest packet with a content name $c_{k}$ from node $n_{j}$, a node $n_{i}$ needs to wait for a deferred timer $T_{i}^{c_{k}}$ before broadcasting the Interest packet. The deferred timer $T_{i}^{c_{k}}$ is a parameter per Interest rather than per node. Each Interest packet has a different deferred timer. We use $e_{r}$ and $e_{\max }$ to denote the residual energy and initial maximum energy at node $n_{i}$. Given the relative transmission distance between $n_{i}$ and $n_{j}, d_{i j}$, and the maximum transmission range $d_{\max }$, the deferred timer for the Interest forwarding $T_{i}^{c_{k}}$ can be calculated as follows:

$$
T_{i}^{c_{k}}=T_{\text {basic }} \cdot\left(1+\alpha \cdot \frac{e_{\max }-e_{r}}{e_{\max }}+(1-\alpha) \cdot \frac{d_{\max }-d_{i j}}{d_{\max }}\right),
$$

where $T_{\text {basic }}$ is a basic constant time slot for the calculation and $\alpha$ is a weight factor to trade off the effects of residual energy and communication distance in the timer calculation. We have $0 \leq \alpha \leq 1$. When the weight factor $\alpha=0$, further neighbor has a higher probability to be chosen to forward Interest packets. With the increase of the $\alpha$ value, residual energy plays more important role in the deferred timer calculation, which helps balance the energy consumption.

In (1), the calculation of the deferred timer $T_{i}^{\mathcal{C}_{k}}$ is dependent on the relative distance and the residual energy. Here, it is assumed that the relative distance can be obtained by some traditional methods. One light-weight solution is based on the RSSI (Received Signal Strength Indication) parameter to estimate the relative distance. Another more accurate but costly solution is using GPS information to calculate the relative distance. However, the detailed distance calculation is out of scope of this paper.

Regarding the Data packet, the deferred timer $T_{d}^{c_{k}}$ for the Data forwarding with a content name $c_{k}$ can be calculated as follows:

$$
T_{d}^{\mathcal{C}_{k}}=\operatorname{rand}\left[0, T_{\text {basic }}\right] .
$$

Similarly, the deferred timer $T_{d}^{c_{k}}$ is also a parameter per packet rather than per node. Each Data packet has a different deferred timer. The key idea in (2) is that Data packets have higher priority in the forwarding than the Interest packets. Based on this, we give the Data packets shorter deferred timers than the Interest packets. Thus, some Interest packets in the deferred queue may be matched by incoming Data packet with a shorter deferred timer, which may avoid redundant packet forwarding and reduce energy consumption.

4.1.3. Packet Suppression. The deferred timer for the Interest and Data forwarding is a new conception in the NDN 
TABLE 1: Actions when an incoming packet matches a deferred packet.

\begin{tabular}{lcl}
\hline Timer type & $\begin{array}{c}\text { Incoming packet } \\
\text { type }\end{array}$ & Actions \\
\hline Interest & $\begin{array}{c}\text { Data } \\
\text { Interest }\end{array}$ & $\begin{array}{l}\text { Cancel } T_{i}^{c_{k}} \text { and Interest forwarding and then forward the Data based on local PIT table } \\
\text { Cancel } T_{i}^{c_{k}} \text { and Interest forwarding after the number of the same incoming Interests reaches a threshold }\end{array}$ \\
\hline Data & $\begin{array}{c}\text { Data } \\
\text { Interest }\end{array}$ & $\begin{array}{l}\text { Cancel } T_{d}^{c_{k}} \text { and Data forwarding after the number of the same incoming Data packets reaches a threshold } \\
\text { Keep the } T_{d}^{c_{k}} \text { and discard the incoming Interest even though its cache lookup hits successfully }\end{array}$ \\
\hline
\end{tabular}

$n_{2}$ : cancel Interest transmission
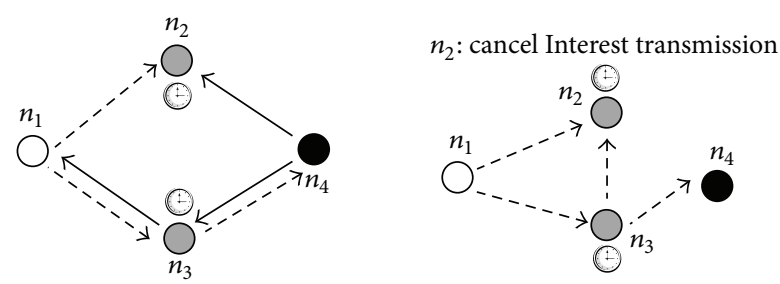

$-\rightarrow$ Interest path

$\longrightarrow$ Data path

FIGURE 3: Interest suppression in flooding mode.

forwarding paradigm. How a NDN router should act after receiving Interest or Data before the deferred timer of the corresponding Interest or Data expires is not defined in the NDN architecture [4] and the NDN forwarding pipeline [24]. In [3, 12], the authors propose an Interest suppression scheme to avoid the waste of bandwidth resources. However, Data suppression is not considered in the literature. Table 1 presents several packet suppression rules to deal with the incoming packet matching with a packet in the deferred waiting queue.

As presented in Table 1, during the waiting time of the Interest deferred timer $T_{i}^{c_{k}}$, if a NDN router overhears the requested Data packet, the timer and the Interest in the waiting queue will be canceled. In the left picture of Figure 3 , a content consumer $n_{1}$ is requesting a content located at node $n_{4}$. Node $n_{2}$ and node $n_{3}$ are two neighbor nodes of node $n_{1}$ and node $n_{4}$. It is assumed that node $n_{3}$ has a shorter deferred timer than node $n_{2}$ for the Interest flooding. And the Data deferred timer is always shorter than the Interest deferred timer. Therefore, the Interest packet may be still deferred in the waiting queue at node $n_{2}$ when the Data packet returns to node $n_{2}$ from node $n_{4}$ in the left picture of Figure 3. In this way, node $n_{2}$ will cancel the Interest transmission in the waiting queue and forward the incoming Data based on local PIT incoming records according to the packet suppression scheme proposed in Table 1. Thus, the Data from the content provider $n_{4}$ will return to the content consumer $n_{1}$ along two reverse paths in Figure 3. In addition, during the $T_{i}^{c_{k}}$ waiting time, if a NDN router overhears the same Interest for specific number of times, $\theta$, the Interest in the waiting queue will be canceled. The threshold of $\theta$ is set to 1 in dense networks according to the literature $[3,12]$. In the right picture of Figure 3, we have the similar application assumption with the left picture. The difference is that the distance between $n_{2}$ : cancel Data transmission
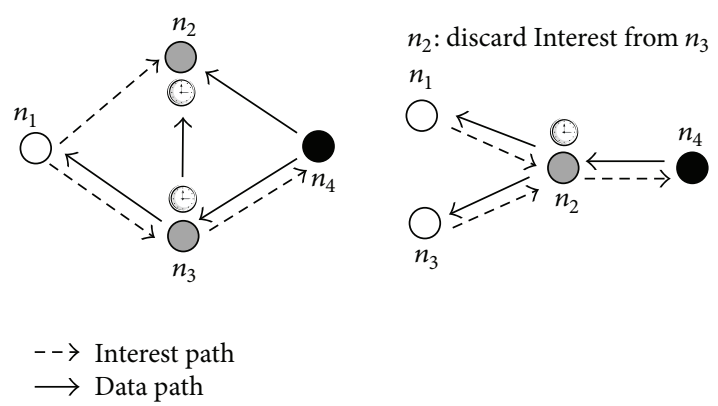

FIGURE 4: Data suppression in flooding mode.

node $n_{2}$ and node $n_{3}$ is closer so that node $n_{2}$ can receive the Interest packet forwarded by node $n_{3}$. In this way, node $n_{2}$ will receive the same Interest query twice from node $n_{1}$ and node $n_{3}$. Therefore, node $n_{2}$ will cancel the Interest transmission in the waiting queue after receiving duplicate Interests from node $n_{3}$ according to Table 1 .

Regarding the Data deferred timer $T_{d}^{c_{k}}$, if a NDN router overhears the same Data for specific number of times, $\theta$, the deferred Data in the waiting queue will be canceled. Similarly, the threshold of $\theta$ can be set to 1 in dense networks. The application assumption in the left picture of Figure 4 is the same as the one in the right picture of Figure 3 . After node $n_{4}$ returns the Data packet, node $n_{2}$ and node $n_{3}$ will receive the Data packet at the same time. It is assumed that node $n_{3}$ has a shorter deferred timer than node $n_{2}$ for the Data flooding. Thus, node $n_{2}$ will receive duplicate Data packets from node $n_{3}$. Then node $n_{2}$ will cancel the deferred Data packet in the waiting queue. In addition, during the $T_{d}^{c_{k}}$ waiting time, the incoming Interest may match the deferred Data packet. In this case, the broadcasting Data packet after the timer expires will meet the request of the incoming Interest. Therefore, the NDN router will discard the newly incoming Interest and will not forward it further. In the right picture of Figure 4, a content consumer $n_{1}$ requested a content located at node $n_{4}$ at first. During the deferred timer of the Data packet at node $n_{2}$, another content consumer $n_{3}$ starts to request the same content matching with the deferred Data at node $n_{2}$. In this case, the incoming Interest from the content consumer $n_{3}$ will be dropped. And the Data packet broadcasted by node $n_{2}$ will reach node $n_{3}$ automatically later.

4.2. FIB Maintenance. The flooding mode of Interest packets is costly in energy consumption, even with the help of the 


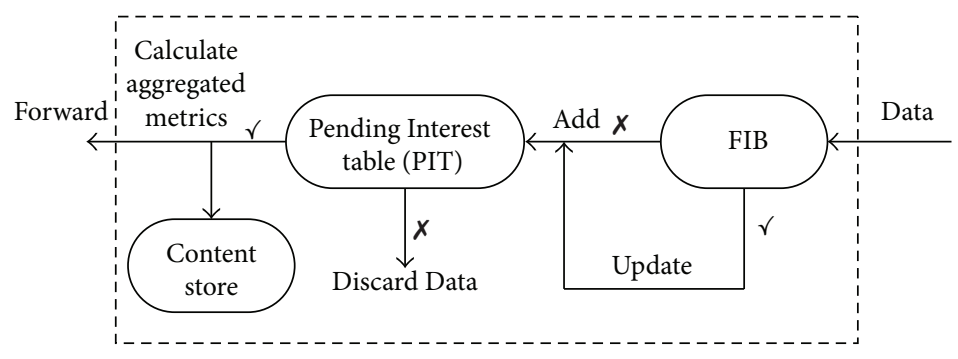

FIGURE 5: NDN data forwarding process with FIB maintenance.

controlled flooding proposed in Section 4.1. Actually, the purpose of the Interest flooding is discovering potential content providers to assist in future Interest forwarding at a lower cost. The key point here is how to dynamically maintain the FIB to guide the Interest forwarding in the DM mode. Inspired by the "breadcrumbs routing" in the literature [23], we propose to maintain the FIB with the passing Data packets in a passive way. The FIB status left by the Data packets along the reverse paths is helpful to speed up the subsequent content discovery in the DM mode at less cost. To support energy efficient Interest forwarding in the DM mode, each entry in the FIB has a format of the following five-tuple $\langle$ Prefix, ForwarderID, Hop, Energy, Timer $\rangle$, indexed by the content name prefix and the ForwarderID.

(i) Prefix: name prefix of the content.

(ii) ForworderID: ID of the node from which the Data comes.

(iii) Hop: hop factor of the path from local node to the content source.

(iv) Energy: residual energy factor of the path from local node to the content source.

(v) Timer: lifetime of this forwarder entry.

In an ad hoc mesh topology, it is possible that broadcasted Interests arrive at the content providers along multiple paths. Original NDN design adopts "one Interest-one Data" mode in which only one Data packet arriving firstly will be accepted finally even if there are more returned Data packets. In order to provide the capability of the selection among more paths in the DM mode, we let the FIB maintain multiple entries for each name prefix to support multipath selection, which is not contradictory with "one Interest-one Data” mode. In the multipath scheme, the Data packets arriving later will be used to update the status of the corresponding FIB entries, even though they cannot be forwarded further due to the missing of the PIT entries.

Therefore, the FIB may maintain multiple entries with different ForwarderIDs for each name prefix. Each entry in the FIB has a timer denoting the lifetime that is reset while receiving corresponding Data packet or Interest packet. A FIB entry will be deleted once the timer expires, which is helpful in deleting the obsolete forwarding information and improving the scalability. In the DM mode, the TTL value will not be reduced in the Interest forwarding in order to improve the efficiency of the content retrieval.
In this paper, each content source replies Data packets carrying initial hop and residual energy information as follows:

$$
\begin{gathered}
h_{\text {source }}=1 ; \\
e_{\text {source }}=\frac{e_{\max }}{e_{r}} .
\end{gathered}
$$

Figure 5 illustrates the extended NDN data forwarding process with FIB maintenance. When a content router $i$ receives a Data packet from an upstream node $j$, it will firstly check whether there has been an entry with the same name prefix and ForwarderID stored in local FIB. If not, the content router $i$ will add a new entry with metrics (denoted as $h(i, j)$ and $e(i, j))$ carried in the incoming Data packet. If yes, the content router will update the metrics of the related entry. Then, the content router will perform normal NDN process. If the PIT lookup hits, the Data packet will be forwarded towards the downstream node(s). Because there may exist multiple entries for the same content prefix in local FIB, Algorithm 1 will be performed to calculate the aggregated hop metric $h(i)$ and energy metric $e(i)$ carried in the Data packet in a probabilistic way.

4.3. Directive Mode (DM). Thanks to the FIB maintenance in Section 4.2, the directive mode can be used to guide the Interests towards the discovered content providers at less cost. As illustrated in Figure 1, the DM mode will be activated once the FIB lookup hits successfully.

Since there may exist multiple entries for the same content prefix in local FIB, the content consumer or intermediate content routers have multiple choices for the neighbor forwarder selection. To balance energy consumption and prolong network lifetime, we propose a least cost forwarding based on the tradeoff between the hop and the energy metrics shown in Algorithm 2.

In Algorithm 2, $\beta$ is a weight factor to balance the tradeoff between total energy consumption and network lifetime. In Section 5, we will observe the impacts of different weight factors on energy efficiency.

Note that the proposed DMIF is only applicable for the Interest forwarding. The Data forwarding process is always the same regardless the selection of Interest forwarding mode. Even the DM mode is chosen, the Data forwarding process is performed in the same way defined in Section 4.2. 
Input: $m$ entries for the same content prefix in the FIB; the hop and the energy metrics of the entry $j$ are denoted as $h(i, j)$ and $e(i, j)$;

(1) Calculate the proportion about the hop and the energy information of each entry;

$$
p \operatorname{hop}(i, j)=\frac{1 / h(i, j)}{\sum_{k=1}^{m} 1 / h(i, k)} ; \quad p_{-} \operatorname{energy}(i, j)=\frac{1 / e(i, j)}{\sum_{k=1}^{m} 1 / e(i, k)}
$$

(2) Calculate the aggregated metrics $h(i)$ and $e(i)$ for hop and energy;

$$
\begin{aligned}
& h(i)=\sum_{k=1}^{m} p \_ \text {hop }(i, k) \cdot h(i, k) ; \\
& e(i)=\sum_{k=1}^{m} p \_\operatorname{energy}(i, k) \cdot e(i, k)
\end{aligned}
$$

Algorithm 1: Aggregated metric calculation at node $i$.

Input: $m$ entries for the same content prefix in the FIB; the hop and the energy metrics of the entry $j$ are denoted as $h(i, j)$ and $e(i, j)$;

(1) Calculate the aggregated metric $m(i, j)$ for each entry; $m(i, j)=\beta \cdot e(i, j)+(1-\beta) \cdot h(i, j), \quad 0 \leq \beta \leq 1$

(2) The ForwarderID of the entry $j$ with the least aggregated metric is chosen as the neighbor forwarder:

$$
m(i, j)=\min m(i, k), \quad k \in[1, k]
$$

Algorithm 2: Least cost Interest forwarding at node $i$.

4.4. Routing Loop Consideration. Thanks to the Nonce field carried in the Interest packet of the NDN, the Interest forwarding is naturally routing loop free. In detail, every Interest carries a random Nonce and each node receiving the Interest can remember the seen Nonces at the PIT table. The incoming Interest with a duplicate Nonce field will be dropped to avoid the routing loop during the Interest forwarding.

According to original design of the NDN, the Data forwarding will not loop since it is performed along the reverse paths of the Interest from upstream nodes to downstream nodes. However, something new needs to be considered in wireless environment with multipath capability. First, each node has only single wireless interface to send and receive the NDN packets. Therefore, there is no way to discriminate the conceptions about upstream nodes or downstream nodes in wireless environment. Second, multipath capability requires a node to receive and process multiple Data packets from different neighbors to remember multiple path information, even though only the firstly arriving Data is forwarded. In addition, there is no any information about the sender ID carried in the Data packet in original NDN design. In this case, the sender of a Data packet may receive the same Data and store the information from its neighbors, thus causing routing loop problem.

For example, in Figure 6, node $n_{3}$ receives a Data packet from node $n_{5}$. It is assumed that the PIT lookup hits locally at node $n_{3}$ and it will forward the Data packet through the wireless broadcast link. In this case, not only the downstream node $n_{2}$ but also the upstream node $n_{5}$ can receive the Data

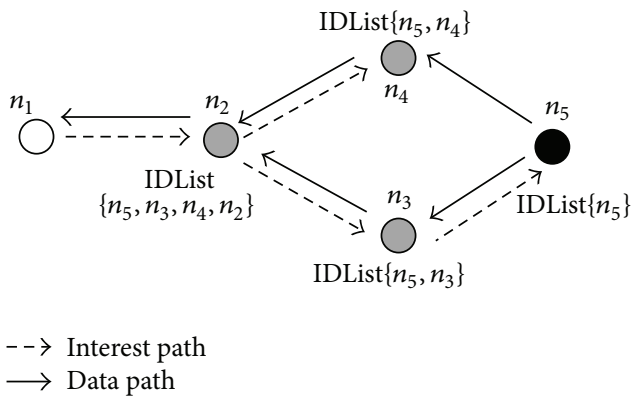

FIGURE 6: Routing loop avoidance in the Data forwarding.

packet from node $n_{3}$. In other words, node $n_{5}$ receives the same Data packet sent by itself before and routing loop happens.

To address the routing loop problem of the Data forwarding, we add a new field named IDList in the Data packet. The IDList contains the IDs of all nodes along the forwarding path of the Data packet. Upon receiving a Data packet, the node will firstly check whether its ID has been listed in the IDList. If yes, routing loop happens and the incoming Data will be dropped. If no, the path information carried in the incoming Data will be used to update local FIB as described in Section 4.2. Figure 6 presents an example to illustrate how the IDList works. In Figure 6 , node $n_{5}$ will discard the Data packet from node $n_{3}$ since its ID is listed in the IDList. Similar routing loop detection is performed at the rest nodes to avoid routing loop in the Data forwarding. The IDList scheme may 


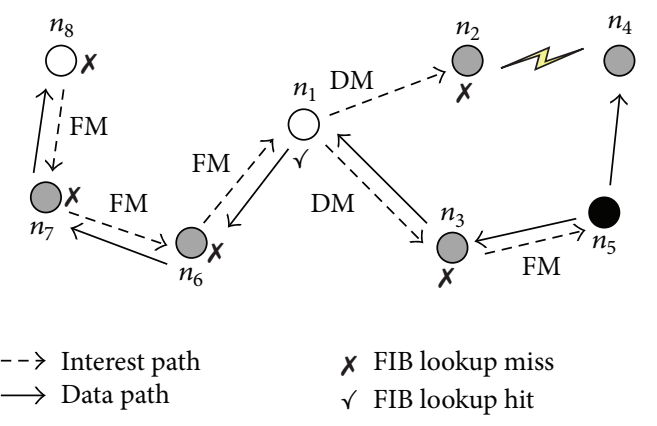

Figure 7: An example for mode shift between the FM and the DM.

not scale well in large scale networks. More efficient routing loop detection schemes may be investigated as future work.

4.5. Example for Mode Shift in the DMIF. Figure 7 provides an example to illustrate how the mode shift works between the FM mode and the DM mode to improve the Interest forwarding. In Figure 7, it is assumed that node $n_{1}$ queried the content located at node $n_{5}$ previously along the path $n_{1}-n_{3}-$ $n_{5}$. According to the FIB maintenance rules, a trail of "breadcrumbs" is left along the reverse path. Now, node $n_{8}$ is querying the same content. Based on the proposed DMIF scheme, the Interest is firstly forwarded in the FM mode until it arrives at node $n_{1}$. After the FIB lookup hits successfully at node $n_{1}$, the Interest is broadcasted in the DM mode with the ForwarderID set as node $n_{3}$. Let us assume that the FIB entry at node $n_{3}$ was deleted due to some reasons. Thus, the forwarding mode will shift back to the FM mode at node $n_{3}$, which broadcasts the Interest to the final destination. With the shift between the FM mode and the DM mode, nodes $n_{2}$ and $n_{4}$ will not be involved in the Interest forwarding originated by node $n_{8}$, which helps reduce the resource consumption and improve the network efficiency.

\section{Performance Evaluation}

5.1. Simulation Settings. This section evaluates the performance of the Interest forwarding scheme DMIF implemented in ndnSIM $2.0[19,20]$, which is extended to support wireless multihop communication and the forwarding strategies proposed in this paper.

In the simulation experiments, there are 100 sensor nodes deployed in a $400 \mathrm{~m} \times 400 \mathrm{~m}$ area in Figure 8 in which all the black nodes on the border are chosen as the content producers that generate the raw data about temperature within its subarea every minute. For example, the raw data name generated by node $n_{0}$ at 16:40 on December 4,2015 , is "/type/temperature/space/area0/time/2015/06/04/1000," according to the naming scheme in Section 3. The white nodes in Figure 8 are chosen as the sink nodes (content consumers) that send periodic Interests to query the interested content about several subareas every five minutes. Every time, 12 interested areas are randomly chosen among the 36 content producers. The grey nodes are NDN routers that can relay packet and cache contents as specified in the NDN. The
TABLE 2: Simulation parameters.

\begin{tabular}{lc}
\hline Parameter & Value \\
\hline Interest size & 50 bytes \\
Data size & 100 bytes \\
$T_{\text {basic }}$ & $7 \mathrm{~ms}$ \\
Wireless interface & IEEE 802.15 .4 \\
Radio coverage range & $100 \mathrm{~m}$ \\
Grid topology size & $400 \times 400$ \\
Grid topology step & $45 \mathrm{~m}$ \\
Number of nodes & 100 \\
Number of content producers & 12 out of 36 \\
Number of content consumers & $1-5$ \\
Initial energy & 5 Joule \\
Energy consumption per bit $e$ & $0.5 \mu \mathrm{J} / \mathrm{bit}$ \\
Simulation time & $3600 \mathrm{~s}$ \\
\hline
\end{tabular}

number of the sinks is changed from 1 to 5 in the experiments. For example, at 16:40 on December 4, 2015, a sink sends the following Interests to query the temperature about subarea0 in the past five minutes:

\begin{tabular}{|l|}
\hline WSNs/type/temperature/space/area0/time/2015/12/04/996 \\
WSNs/type/temperature/space/area0/time/2015/12/04/997 \\
WSNs/type/temperature/space/area0/time/2015/12/04/998 \\
WSNs/type/temperature/space/area0/time/2015/12/04/999 \\
WSNs/type/temperature/space/area0/time/2015/12/04/1000
\end{tabular}

In the experiments, it is assumed that each node sends and receives packets with fixed transmission and reception power. Therefore, the energy consumption is independent of the transmission distance through a wireless link. Based on this, we adopt the following energy model $[25,26]$ to evaluate the power consumption in the simulation experiments:

$$
c \approx e \cdot\left(b_{r}+b_{t}\right)
$$

where $c$ denotes the total energy consumption of one node for receiving $b_{r}$ bits and transmitting $b_{t}$ bits and $e$ is a factor indicating the energy consumption per bit at the receiver circuit.

In order to support more efficient forwarding, we let the FIB maintain multiple entries and update the metrics dynamically, which may bring more cost in the FIB memory and energy consumption. The ndnSIM platform used in this paper can simulate almost real experiment environment because it adopts NFD (Named Data Networking Forwarding Daemon) as the forwarding kernel. In other words, the extra overhead caused by supporting multipath capability can be included in the energy consumption automatically.

The simulation parameters are listed in Table 2.

5.2. Simulation Methodology. As analyzed in Section 2, the literature $[3,12]$ proposes two Interest forwarding strategies including blind forwarding (BF-RD) and provider-aware forwarding (PAF), which are chosen as the baseline schemes for comparison in the simulation experiments.

(i) BF-RD: the Interest is always forwarded in the flooding mode based on a randomly deferred timer. 


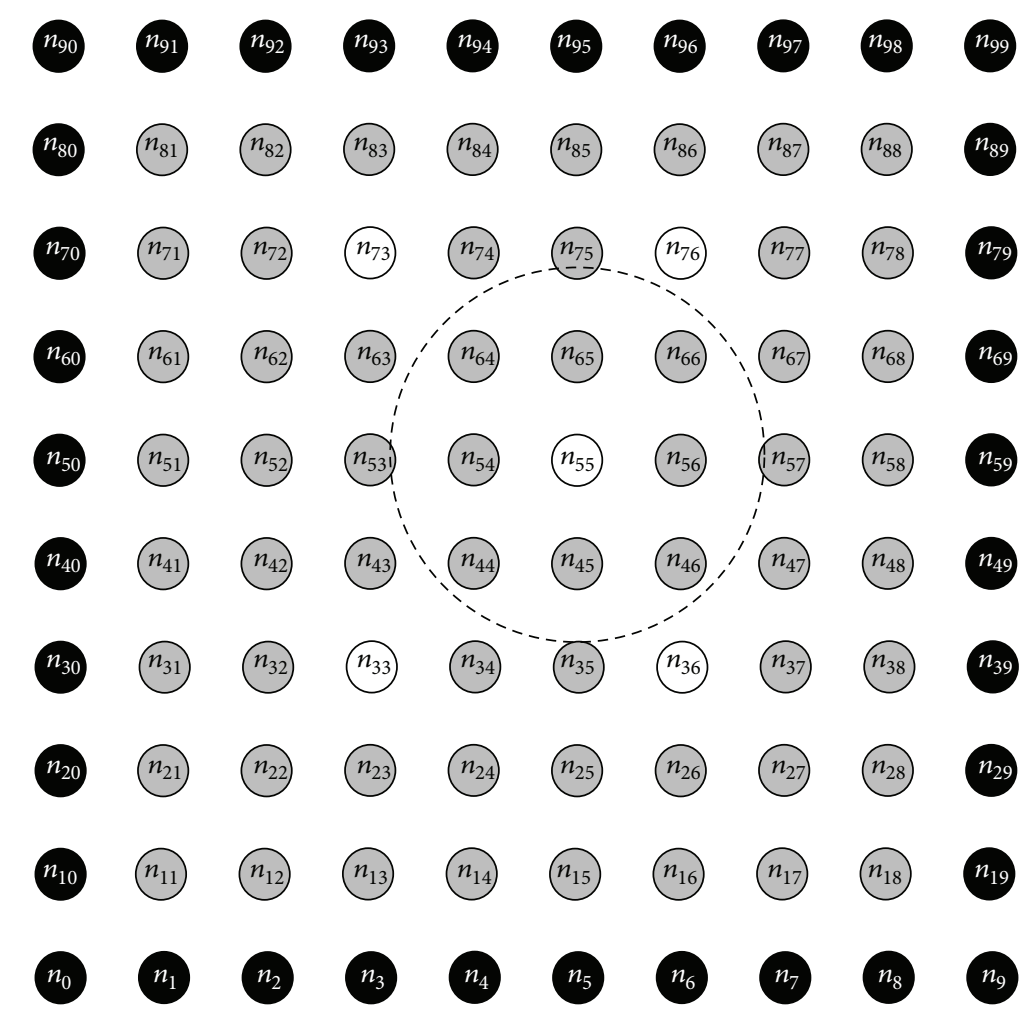

FIGURE 8: Simulation scenario: grid topology.

(ii) PAF: the content consumer includes the information about the nearest provider into the Interest so that it can be forwarded to specific direction after the BF-RD is performed.

In this paper, we propose an energy efficient strategy named DMIF, which consists of a flooding mode and a directive mode for Interest forwarding. For more intuitive comparison with the baseline schemes, we name the flooding mode in the DMIF as BF-ED correspondingly due to its energy efficient deferred timer calculation. In the BF-ED, the Interest is always forwarded in the flooding mode based on a deferred timer proposed in (1). In addition, the other energy efficient schemes including scope control and packet suppression presented in Section 4.1 are also used in the BF-ED. In other words, the BF-ED is a subset of the DMIF scheme.

We observe the following metrics to evaluate the performance of the DMIF scheme.

(i) Total energy consumption is total energy consumed by all sensor nodes.

(ii) Energy equilibrium rate (EER) is defined as the variance of the energy consumption of each node. The EER denotes the balance of the energy consumption of the whole network and reflects the network lifetime indirectly.

(iii) Hop count is the average hop count of the content retrieval for each Interest request. (iv) Network lifetime is defined as the time length from the beginning of the simulation to the first node's energy exhaustion.

\subsection{Simulation Results}

5.3.1. Performance of BF-ED. First, we evaluate the impacts of the weight factor $\alpha$ in (1) on the performance of the BF-ED.

In Figures 9 and 10, when the weight factor $\alpha=0$, only the distance value between adjacent nodes is considered in the deferred timer calculation. In this way, further neighbor has a higher probability to be chosen to forward Interest packets during the blind flooding, which will help reduce the hop count and the energy consumption. With the increase of the $\alpha$ value, the distance between adjacent nodes plays less important role in calculating the Interest deferred timer and then the total energy consumption and average hop count rises gradually. What is more important is the impacts of the $\alpha$ on energy equilibrium rate shown in Figure 11, in which the energy equilibrium rate decreases with the increase of the $\alpha$ value. The key idea of (1) is trying to balance the energy consumption by adjusting the weight factor $\alpha$. In the proposed BF-ED, bigger $\alpha$ value denotes that residual energy plays more important role in the deferred timer calculation. In other words, choosing the node with more residual energy to forward Interests will help balance the energy consumption, which is consistent with the results in Figure 11.

In Figures 12, 13, and 14, we compare the performance of the proposed BF-ED with the baseline scheme BF-RD $[3,12]$ 


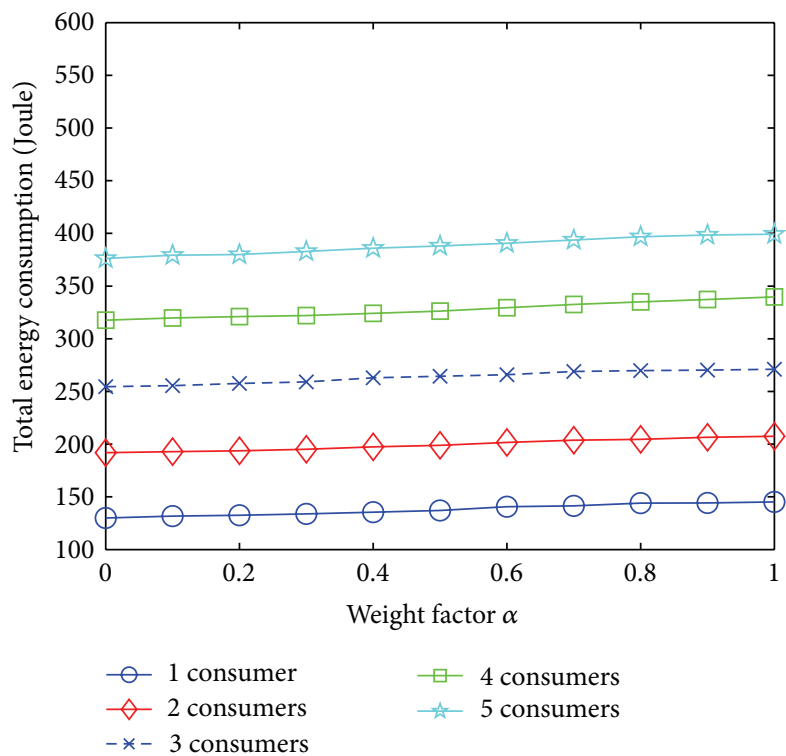

Figure 9: BF-ED: impact of weight factor $\alpha$ on total energy consumption.

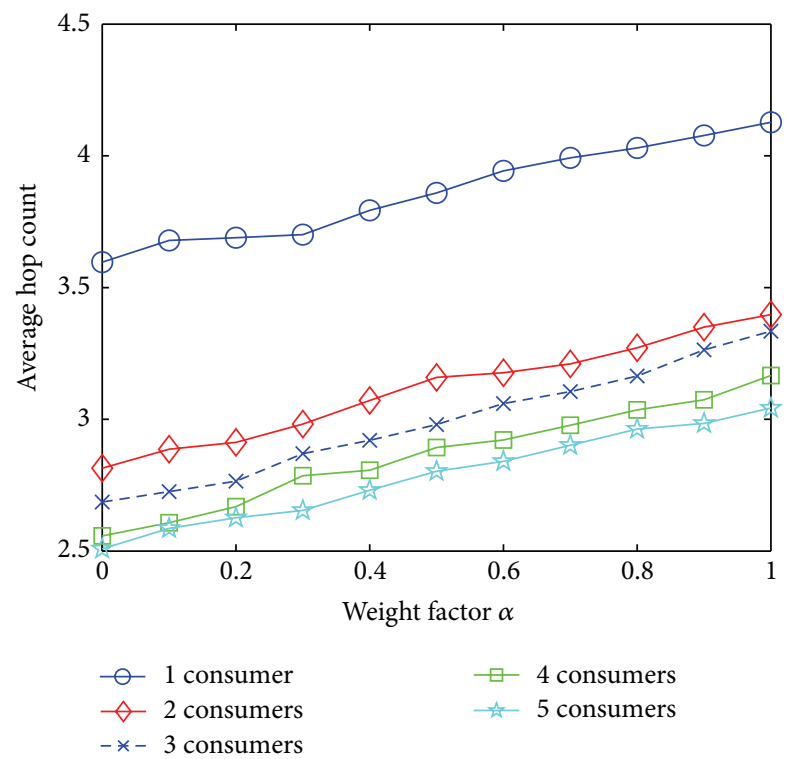

FIGURE 10: BF-ED: impact of weight factor $\alpha$ on average hop count.

by changing the number of the content consumers from 1 to 5 . More content consumers means more Interest requests consuming more energy in Figure 12 and then causing bigger variance of the energy consumption in Figure 14. Note that the experiments with more consumers exhibit better performance than the ones with less consumers in terms of average hop count in Figure 10, thanks to in-network caching adopted in the NDN architecture. It can be observed that the BF-RD exhibits medium performance compared with two border conditions under the BF-ED with $\alpha=0$ and $\alpha=1$. In a summary, the weight factor $\alpha$ has important impacts on the performance of the BF-ED scheme. In practice, a suitable $\alpha$ value needs to be chosen to trade off the energy

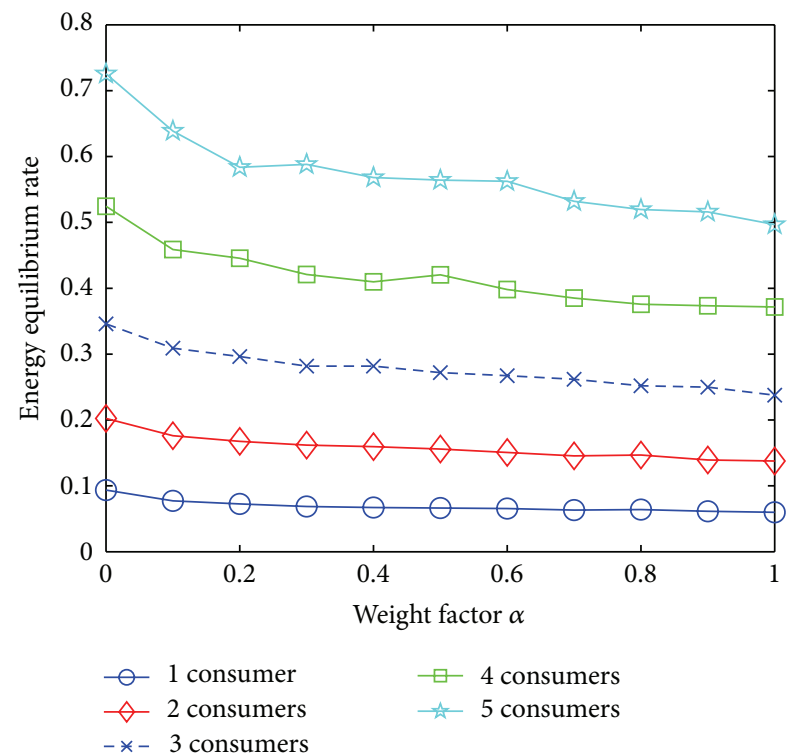

FIGURE 11: BF-ED: impact of weight factor $\alpha$ on energy equilibrium rate.

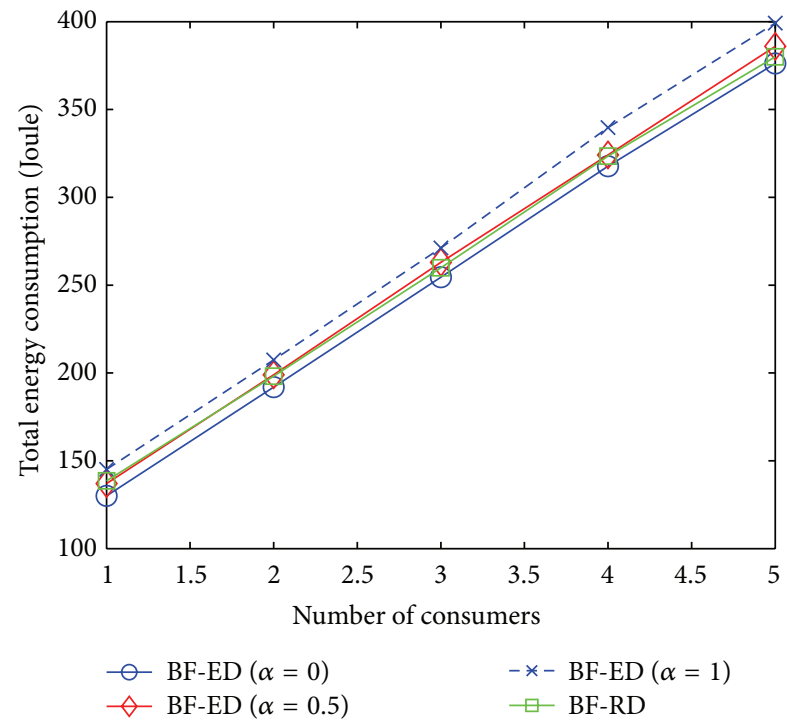

Figure 12: Comparison between BF-ED and BF-RD: total energy consumption.

consumption and the energy equilibrium rate according to actual application requirements. For simplicity, the $\alpha$ value is chosen as 0.5 in the following experiments.

5.3.2. Performance of DMIF. Figures 15 and 16 show the impacts of the weigh factor $\beta$ in Algorithm 2 on total energy consumption and average hop count. It can be observed that the weigh factor $\beta$ exhibits similar impacts with the weight factor $\alpha$ as presented in Section 5.3.1. With the rise of the $\beta$ value, the hop count plays less important roles in choosing the Interest forwarder, which results in the increase of total energy consumption and average hop count for content retrieval. On the other hand, with the change of the $\beta$ value 


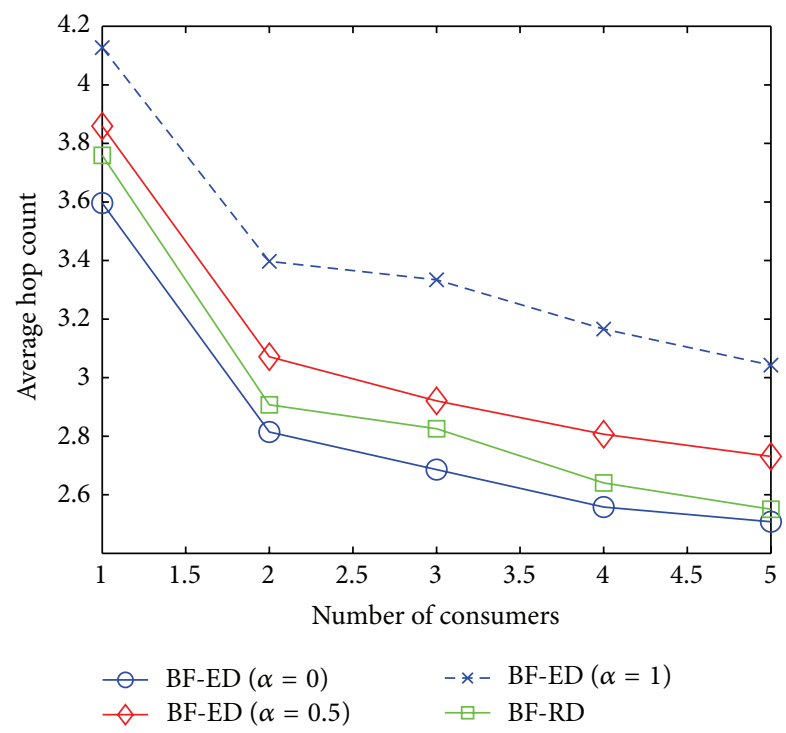

FIGURE 13: Comparison between BF-ED and BF-RD: average hop count.

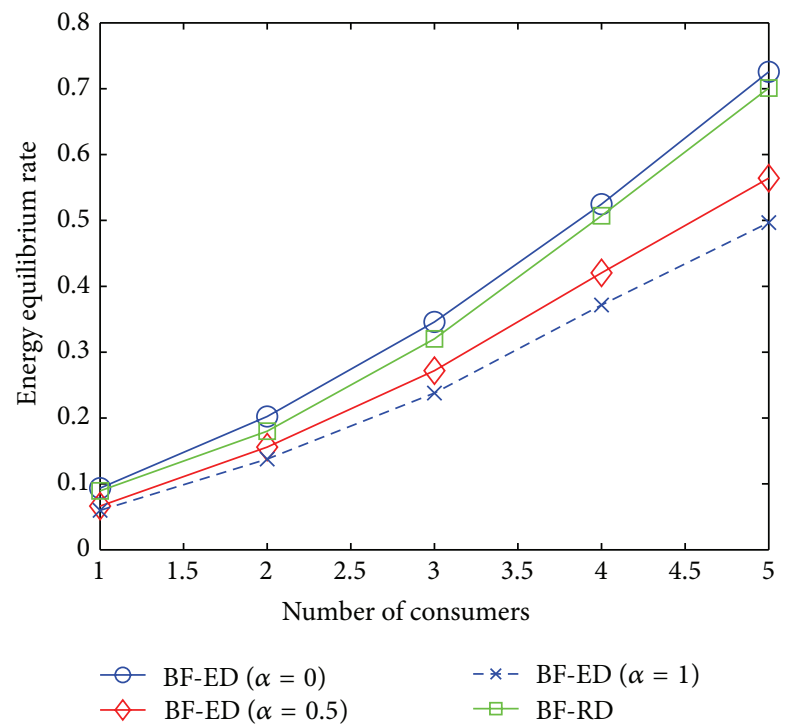

FIGURE 14: Comparison between BF-ED and BF-RD: energy equilibrium rate.

from 0 to 1 , the content consumers and the intermediate nodes would rather choose the neighbor with higher residual energy as the Interest forwarder. In other words, the residual energy outperforms the hop count in Algorithm 2 with the increase of the $\beta$ value, which helps balance the energy consumption from the viewpoint of the whole network and leads to the descending trend of the energy equilibrium rate in Figure 17.

Next, we compare the performance of the proposed DMIF and the PAF by changing the number of the content consumers from 1 to 5. In Figure 18, the total energy consumption in both the DMIF and the PAF increases when more content consumers send more Interests to request the contents. In contrast with the PAF, the DMIF has better

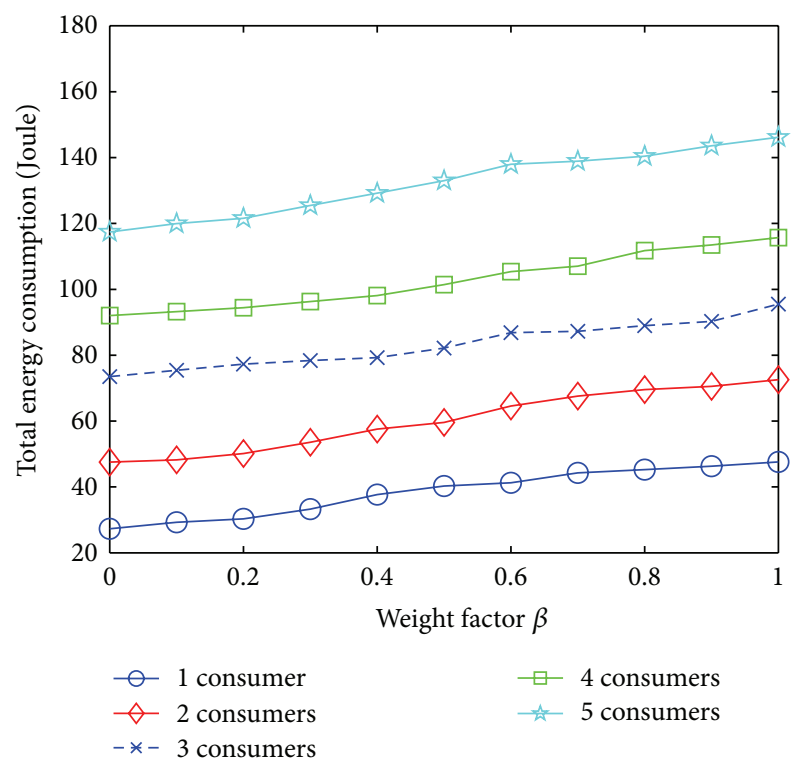

FIgURE 15: DMIF: impact of weight factor $\beta$ on total energy consumption $(\alpha=0.5)$.

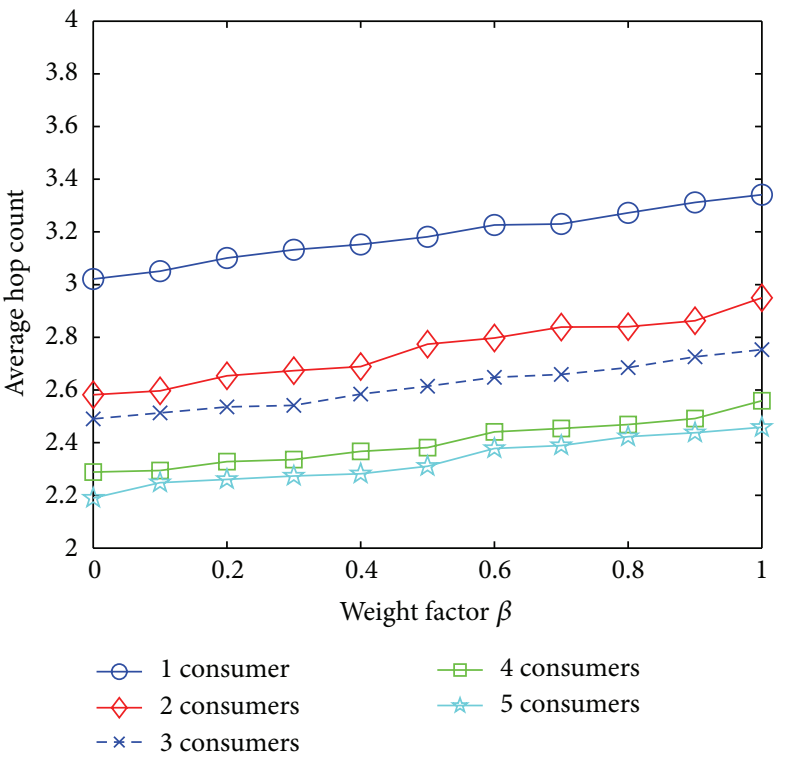

FIGURE 16: DMIF: impact of weight factor $\beta$ on average hop count $(\alpha=0.5)$.

performance in terms of total energy consumption, since the DMIF supports flexible mode shift for the Interest forwarding according to the results of the FIB lookups. In the PAF scheme, the blind flooding phase and the provider awareness phase are two parallel phases. The information about the content provider carried in the Interest is only added by the content consumer after the blind flooding phase. In this case, the blind flooding mode will continue till the content providers even though an intermediate node has already FIB information for the requested content. When the PAF forwarding fails due to the miss of the FIB lookup, the content consumer needs to restart the flooding phase throughout the 


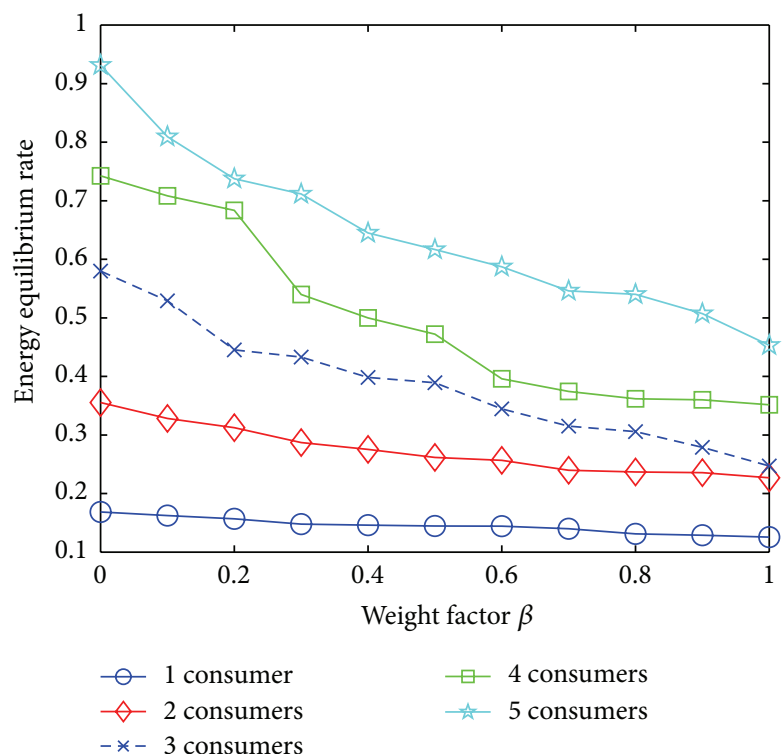

FIGURE 17: DMIF: impact of weight factor $\beta$ on energy equilibrium rate $(\alpha=0.5)$.

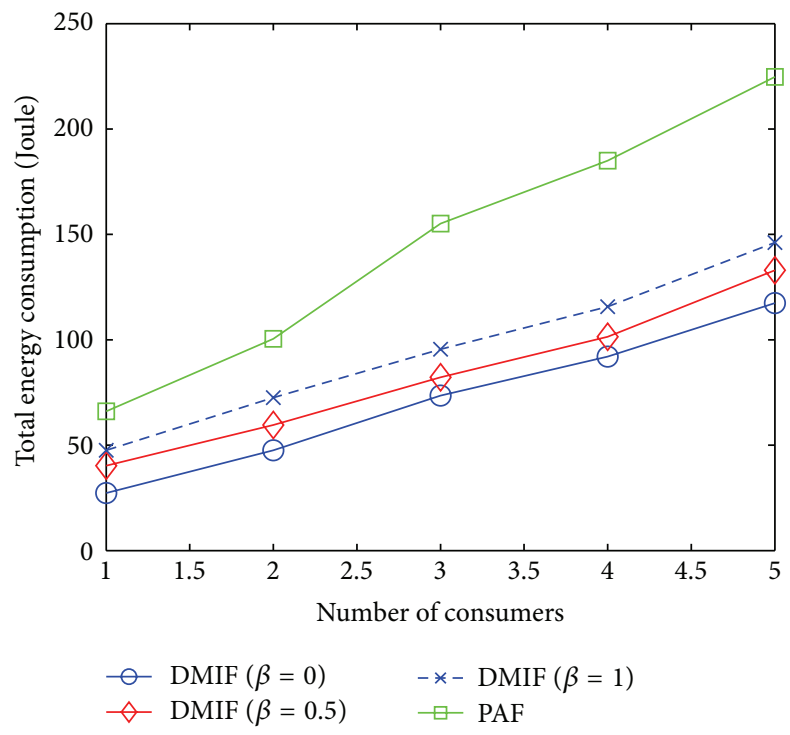

FIgURE 18: Comparison between DMIF and PAF: total energy consumption $(\alpha=0.5)$.

whole network after the Interest times out. In the proposed DMIF, the directive mode can shift to the flooding mode locally, which helps reduce the energy consumption greatly in Figure 18.

As analyzed in Section 5.3.1, the requested Interests may be satisfied by the intermediate nodes rather than the content providers, thanks to the in-network cache technology in the NDN architecture. Therefore, the average hop count for the content retrieval drops with the increase of the number of the content consumers in Figure 19. It also can be observed that the average hop count in the PAF is shorter than the proposed DMIF, since the content consumers always choose the nearest

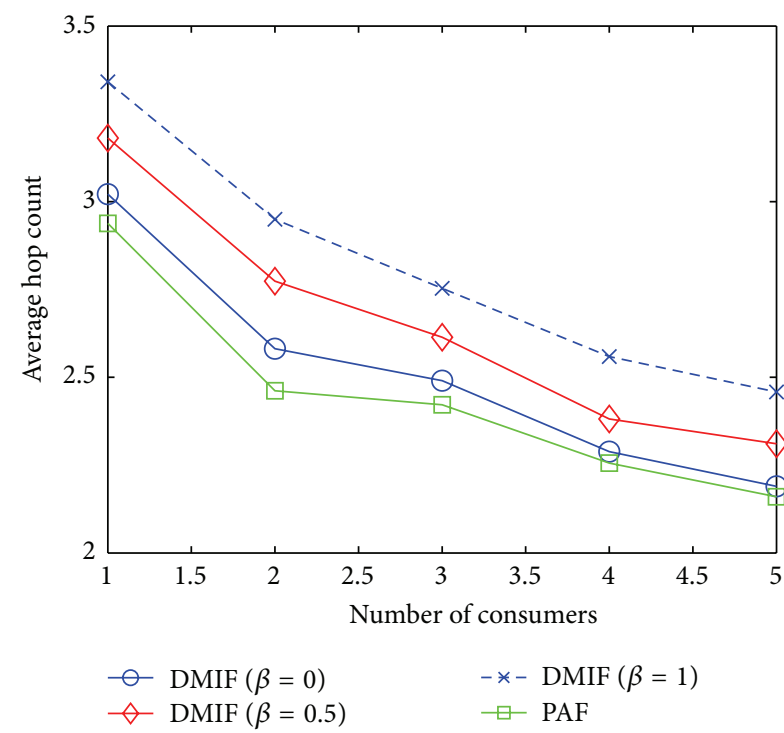

FIGURE 19: Comparison between DMIF and PAF: average hop count $(\alpha=0.5)$.

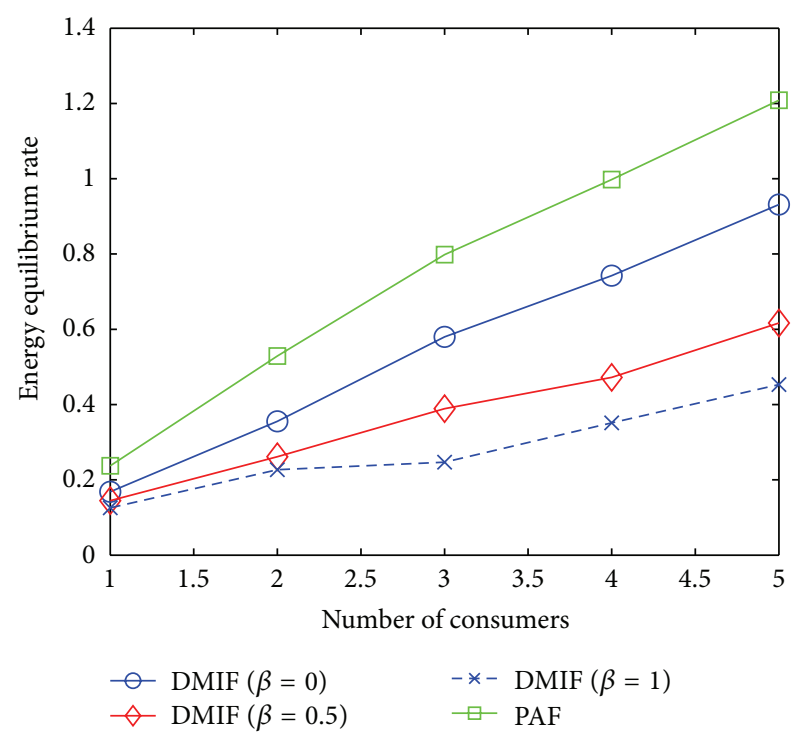

Figure 20: Comparison between DMIF and PAF: energy equilibrium rate $(\alpha=0.5)$.

provider as its destination in the PAF. In the DMIF, the weight factor $\beta=0$ means only hop count is considered in choosing the Interest forwarder. However, the DMIF with $\beta=0$ still has a little more average hop count than the PAF, because the hop count calculation in Algorithm 1 is based on the weights of all hop metrics.

In Figure 20, the proposed DMIF gains better energy equilibrium rate and more balanced energy consumption than the PAF due to the following three reasons: (1) the average energy consumption in the DMIF is lower than the one in the PAF; (2) the metric aggregation in Algorithm 1 is based on weights of the metrics from each neighbor; (3) the weight factor $\beta$ helps avoid choosing the nodes with little 


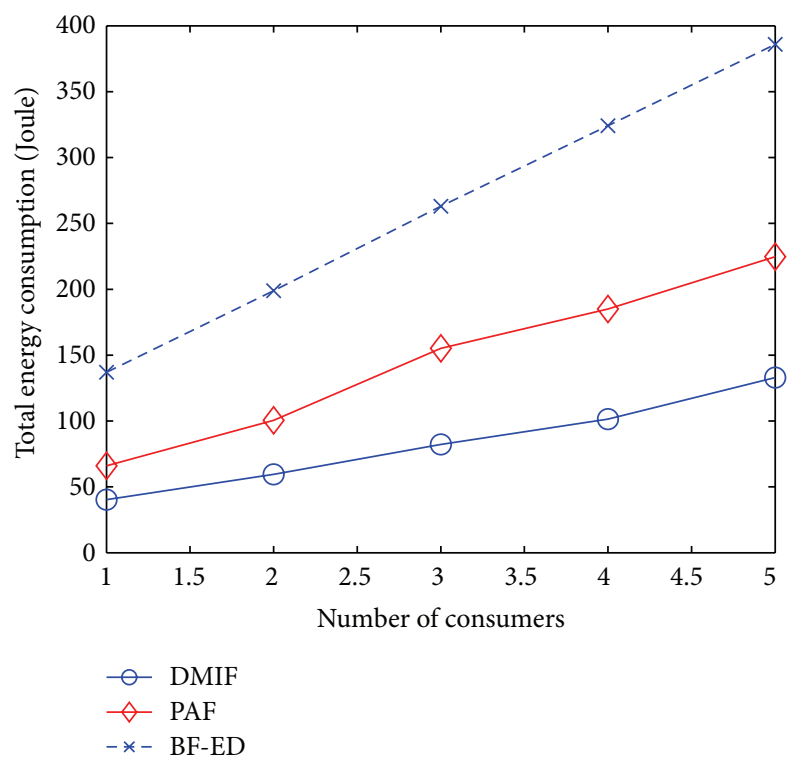

Figure 21: Comparison between DMIF and BF-ED: total energy consumption $(\alpha=0.5 ; \beta=0.5)$.

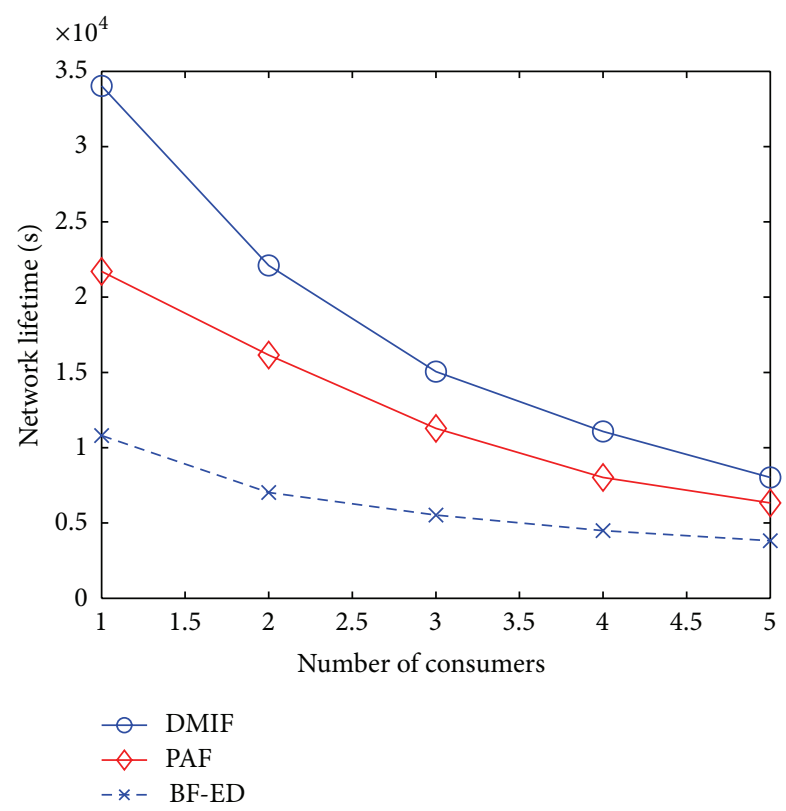

FIgUre 22: Comparison between DMIF and BF-ED: network lifetime $(\alpha=0.5 ; \beta=0.5)$.

residual energy for the Interest forwarding. On the other hand, there is no consideration about flexible mode shift and energy consumption balance in the PAF, thus leading to higher energy equilibrium rate than the DMIF.

5.3.3. Comparison between DMIF and BF-ED. Finally, we compare the DMIF and the BF-ED proposed in this paper in terms of total energy consumption and network lifetime in Figures 21 and 22, in which the weight factors $\alpha$ and $\beta$ are both set as 0.5 . Here, the network lifetime is defined as the time length from the beginning of the simulation to the first node's energy exhaustion. Actually, the information about Figure 21 has been presented in Figures 12 and 18. In this section, we make an intuitive figure to illustrate the advantage of the DMIF about total energy consumption. It can be observed that the BF-ED with only flooding mode has the most energy consumption and the shortest network lifetime. With the help of the flexible mode shift and energy aware neighbor selection, the DMIF scheme can reduce the total energy consumption and prolong the network lifetime significantly compared with the BF-ED and the PAF in Figures 21 and 22.

\section{Conclusion}

In this paper, we propose an energy efficient Interest forwarding scheme called DMIF for NDN-based WSNs. In the DMIF, two combined forwarding modes (flooding mode and directive mode) can be shifted flexibly based on the FIB lookup results so that the network overhead can be reduced greatly. In addition, several energy efficient mechanisms including flooding scope control, broadcast storm avoidance, packet suppression, and energy weight factors are proposed to save and balance the energy consumption. Simulation experiments under the ndnSIM show that the DMIF scheme outperforms the baseline schemes in terms of total energy consumption, energy equilibrium rate, and network lifetime.

As future work, we plan to conduct theoretical analysis and real experiments to evaluate the proposed schemes.

\section{Competing Interests}

The authors declare that they have no competing interests.

\section{Acknowledgments}

This work is supported by the National Basic Research Program of China (973 Program) under Grant no. 2013CB329100, the Fundamental Research Funds for the Central Universities under Grant no. 2015JBM004, the National High Technology Research and Development Program (863) of China under Grant no. 2015AA011906, Beijing Nova Program under Grant no. Z151100000315052, Beijing Higher Education Young Elite Teacher Project under Grant no. YETP0532, and the National NSF of China under Grants nos. 61100217 and 61232017.

\section{References}

[1] K. Akkaya and M. Younis, "A survey on routing protocols for wireless sensor networks," Ad Hoc Networks, vol. 3, no. 3, pp. 325-349, 2005.

[2] J. N. Al-Karaki and A. E. Kamal, "Routing techniques in wireless sensor networks: a survey," IEEE Wireless Communications, vol. 11, no. 6, pp. 6-28, 2004.

[3] M. Amadeo, C. Campolo, A. Molinaro, and N. Mitton, "Named data networking: a natural design for data collection in wireless sensor networks," in Proceedings of the IFIP Wireless Days Conference (WD '13), pp. 1-6, Valencia, Spain, November 2013.

[4] L. Zhang, A. Afanasyev, J. Burke et al., "Named data networking," ACM SIGCOMM Computer Communication Review, vol. 44, no. 3, pp. 66-73, 2014. 
[5] M. Amadeo, C. Campolo, A. Molinaro et al., "Multi-source data retrieval in IoT via named data networking," in Proceedings of the 1st International Conference on Information-Centric Networking (ICN '14), pp. 67-76, Paris, France, September 2014.

[6] E. Baccelli, C. Mehlis, O. Hahm, T. C. Schmidt, and M. Wählisch, "Information centric networking in the IoT: experiments with NDN in the wild," in Proceedings of the 1st ACM International Conference on Information-Centric Networking (ICN '14), pp. 77-86, ACM, September 2014.

[7] C. Wang, J. Li, F. Ye, and Y. Yang, "NETWRAP: an NDN based real-timewireless recharging framework for wireless sensor networks," IEEE Transactions on Mobile Computing, vol. 13, no. 6, pp. 1283-1297, 2014.

[8] Z. Ren, M. A. Hail, and H. Hellbruck, "CCN-WSN-a lightweight, flexible Content-Centric Networking protocol for wireless sensor networks," in Proceedings of the IEEE 8th International Conference on Intelligent Sensors, Sensor Networks and Information Processing (ISSNIP '13), pp. 123-128, Melbourne, Australia, April 2013.

[9] M. Amadeo, C. Campolo, A. Iera, and A. Molinaro, "Named data networking for IoT: an architectural perspective," in Proceedings of the European Conference on Networks and Communications (EuCNC '14), pp. 1-5, Bologna, Italy, June 2014.

[10] M. Amadeo, C. Campolo, A. Molinaro, and G. Ruggeri, "Content-centric wireless networking: a survey," Computer Networks, vol. 72, pp. 1-13, 2014.

[11] C. Intanagonwiwat, R. Govindan, and D. Estrin, "Directed diffusion: a scalable and robust communication paradigm for sensor networks," in Proceedings of the 6th Annual International Conference on Mobile Computing and Networking (MobiCom '00), pp. 56-67, ACM, 2000.

[12] M. Amadeo, C. Campolo, and A. Molinaro, "Forwarding strategies in named data wireless ad hoc networks: design and evaluation," Journal of Network and Computer Applications, vol. 50, pp. 148-158, 2015.

[13] L. Wang, A. Afanasye, R. Kuntz, R. Vuyyuru, R. Wakikawa, and L. Zhang, "Rapid traffic information dissemination using named data," in Proceedings of the 1st ACM Workshop on Emerging Name-Oriented Mobile Networking Design-Architecture, Algorithms, and Applications (NoM '12), pp. 7-12, 2012.

[14] L. You, B. Zhou, L.-C. Tung, M. Gerla, A. Ramesh, and L. Nagaraja, "Energy-efficient content retrieval in mobile cloud," in Proceedings of the 2nd ACM SIGCOMM Workshop on Mobile Cloud Computing (MCC '13), pp. 21-26, ACM, Hong Kong, August 2013.

[15] Y.-T. Yu, R. B. Dilmaghani, S. Calo, M. Y. Sanadidi, and M. Gerla, "Interest propagation in named data manets," in Proceedings of the International Conference on Computing, Networking and Communications (ICNC '13), pp. 1118-1122, IEEE, San Diego, Calif, USA, January 2013.

[16] F. Angius, M. Gerla, and G. Pau, "BLOOGO: BLOOm filter based GOssip algorithm for wireless NDN," in Proceedings of the 1st ACM Workshop on Emerging Name-Oriented Mobile Networking Design-Architecture, Algorithms, and Applications (NoM '12), pp. 25-30, 2012.

[17] M. Amadeo, C. Campolo, and A. Molinaro, "Enhancing content-centric networking for vehicular environments," Computer Networks, vol. 57, no. 16, pp. 3222-3234, 2013.

[18] M. Amadeo, A. Molinaro, and G. Ruggeri, "E-CHANET: routing, forwarding and transport in Information-Centric multihop wireless networks," Computer Communications, vol. 36, no. 7, pp. 792-803, 2013.
[19] S. Mastorakis, "ndnSIM 2.0: a new version of the NDN simulator for NS-3," Tech. Rep. NDN-0028, NDN, 2015.

[20] A. Afanasyev, I. Moiseenko, and L. Zhang, "ndnSIM 2.0: ndnSIM: NDN simulator for NS-3," Tech. Rep. NDN-0005, NDN, 2012.

[21] M. A. Hail, M. Amadeo, A. Molinaro, and S. Fischer, "On the performance of caching and forwarding in information-centric networking for the IoT,' in Proceedings of the 13th International Conference (WWIC '15), M. C. Aguayo-Torres, G. Gómez, and J. Poncela, Eds., vol. 9071 of Lecture Notes in Computer Science, pp. 313-326, Malaga, Spain, May 2015.

[22] M. Varvello, I. Rimac, U. Lee, L. Greenwald, and V. Hilt, "On the design of content-centric MANETs," in Proceedings of the 8th International Conference on Wireless On-Demand Network Systems and Services (WONS '11), pp. 1-8, Bardonecchia, Italy, January 2011.

[23] E. Rosensweig and J. Kurose, "Breadcrumbs: efficient, besteffort content location in cache networks," in Proceedings of the IEEE Conference on Computer Communications (IEEE INFOCOM '09), pp. 2631-2635, Rio de Janeiro, Brazil, April 2009.

[24] A. Afanasyev, J. Shi, B. Zhang et al., "NFD developer's guide," Tech. Rep. NDN-0021, NDN, 2015.

[25] M. Wang, S. Basagni, E. Melachrinoudis, and C. Petrioli, "Exploiting sink mobility for maximizing sensor networks lifetime," in Proceedings of the 38th Annual Hawaii International Conference on System Sciences, p. 287a, January 2005.

[26] S. Gao, H. Zhang, and S. K. Das, "Efficient data collection in wireless sensor networks with path-constrained mobile sinks," IEEE Transactions on Mobile Computing, vol. 10, no. 4, pp. 592 608,2011 

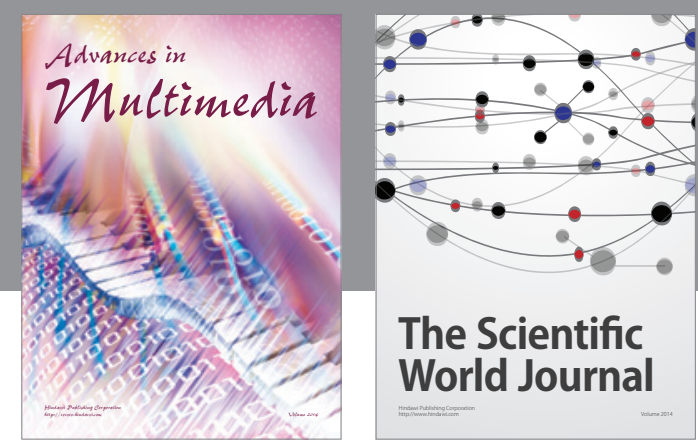

The Scientific World Journal
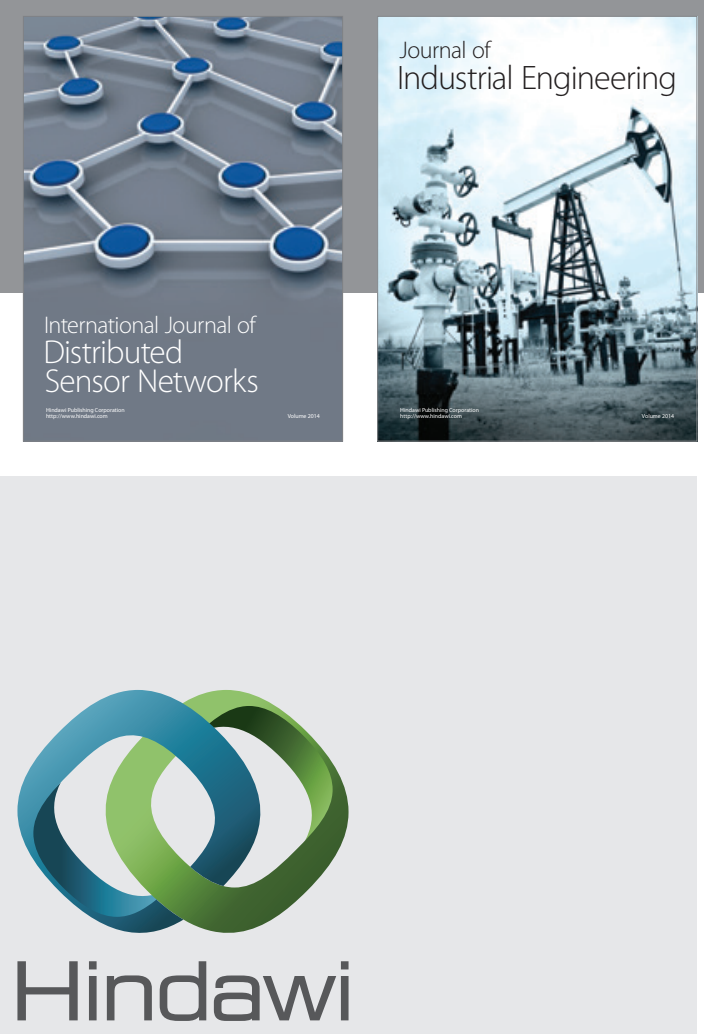

Submit your manuscripts at

http://www.hindawi.com

\section{Computer Networks} and Communications
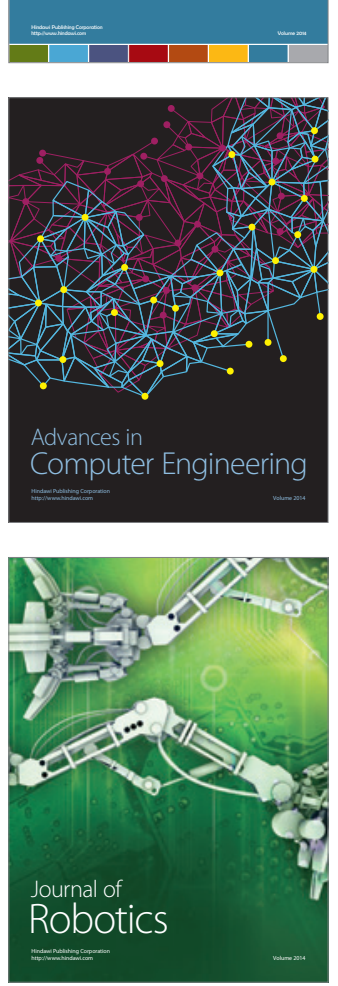
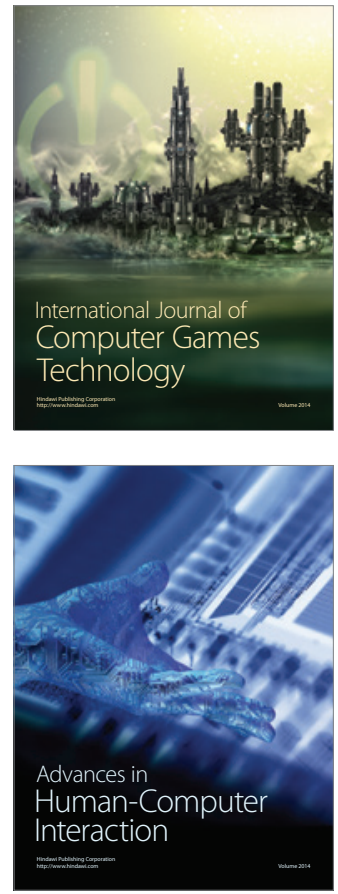
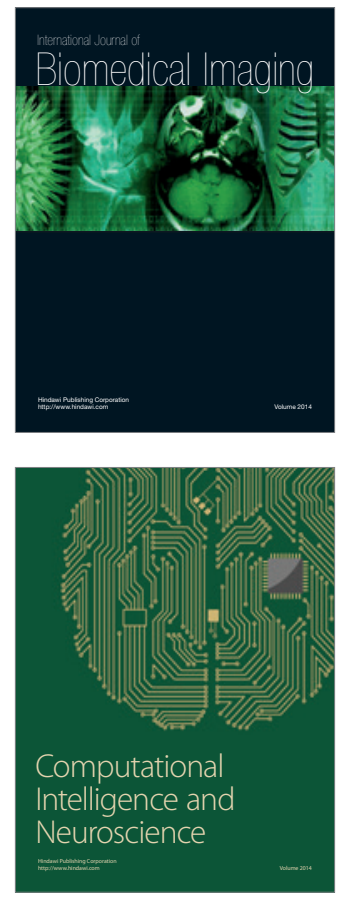
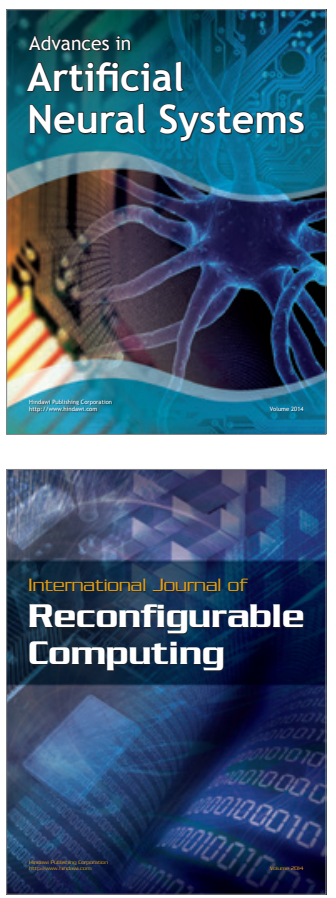
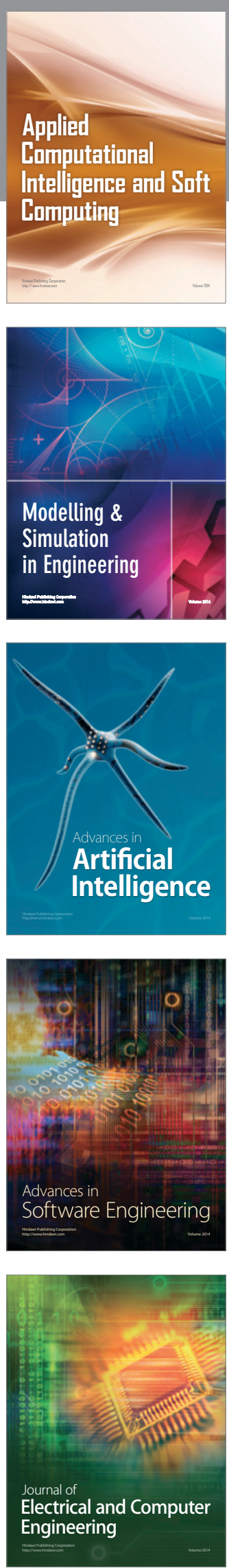